\title{
The Dynamical Connection Between Phaethon and Pallas
}

\author{
Nataša Todorović * \\ Belgrade Astronomical Observatory, Volgina 7, P.O.Box 7411060 Belgrade, Serbia
}

Accepted XXX. Received YYY; in original form ZZZ

\section{INTRODUCTION}

The Near Earth Asteroid (3200) Phaethon was found by the Infrared Astronomical Satellite (Green and Kowal 1983) in October, 1983 and it was identified as the long sought parent body of the Geminid meteor shower (Whipple 1983; Gustafson 1989; Williams 1993). Not long after, it turned out that unlike other meteoroid streams originating from comets, Phaethon, the parent body of the Geminids - is an asteroid.

The activity of this unusual asteroid has been largely studied in the literature. According to Delbo (2014) the large eccentricity of Phaethon's orbit $(e \sim 0.89)$ plays an important role in its geological activity. Periodically approaching to and then distancing from the Sun causes cyclical cooling and heating, leading to the thermal cracking of its mineralogical surface. The intensive solar radiation near perihelion at $q \sim 0.14$ AU ejects away small grain-sized particles that enter into the Earth's atmosphere producing Geminids. However, one of the open questions in this scenario is that the estimated mass loss from Phaethon $\sim 3 \cdot 10^{5} \mathrm{~kg}$ (Jewitt 2013) does not account for the total mass of the Geminids $\sim 10^{12}-10^{13} \mathrm{~kg}$ (Jenniskens 1994).

Spectral attributes of Phaethon (see for example Hanus (2016) and references therein) classified this asteroid as a Btype object (De Meo et al 2009). The largest population of such bodies can be found in the asteroid family Pallas, indicating that Pallas could be the dynamical origin of Phaethon. Also, there should be an efficient dynamical mechanism able to drive bodies from the part of the main

^ E-mail:ntodorovic@aob.rs belt where the Pallas family is located, down to the highly inclined near-Earth region of Phaethon. In de Leon et al (2010), it was illustrated that Phaethon is connected both spectroscopically and dynamically to the Pallas family, and that the major role in this transportation is played by the two strongest mean motion resonances (MMRs) with Jupiter in the region of Pallas family: the 8:3 at $a \sim 2.70 \mathrm{AU}$ and the 5:2 at $a \sim 2.82 \mathrm{AU}$. However, a relatively low fraction of test particles (only $\sim 2 \%$ ) placed into the two resonances where actually able to recover a Phaethon-like orbit.

Our goal is to revisit the role of the 8:3 and 5:2 MMRs in the Pallas-Phaethon dynamical connection. Using sophisticated numerical methods, we illustrate that this dynamical link acts with a much higher efficiency than hitherto observed.

\section{METHOD AND RESULTS}

The standard way to study the dynamics of a resonance is to inject into it a certain number of test objects, clone the particles (with the corresponding standard deviation) using Gaussian distributions in the orbital element space, and integrate them in some fixed time, during which we observe and study their dynamical evolution. In using this method, however, it is very common that only a small fraction of test particles interact with the resonance, despite long computational times.

Here we study the resonant dynamics using a method introduced in Todorović (2017). The first step is to calculate short-term dynamical maps of the resonance using the Fast Lyapunov Indicator - FLI (Froeschlé et al 1997b,a). 
It is important that the calculation times of the maps are short, because in this way the fine structure of chaos in the resonance can be clearly detected (Guzzo and Lega 2014; Todorović 2017). The more unstable parts, easily noticeable on the maps, represent 'good' starting positions for diffusion. Particles chosen along them should be active as soon the integration starts, making this method computationally cheaper and more efficient.

Let us mention that originally, FLI was used for idealized systems such as symplectic maps or simplified Hamiltonians (Froeschlé et al 2000; Lega and Froeschlé 2003; Froeschlé et al 2005; Todorović et al 2008, 2011), but later it was successfully applied to studies of asteroids, planetary and satellite systems as well, from the close neighborhood of Earth (Daquin et al 2016; Rosengren 2015) to the outer Solar System (Guzzo 2005, 2006) or exoworlds (see for example Pilat-Lohinger et al (2002); Dvorak et al (2003); Sándor (2007); Schwarz (2011)).

\subsection{The 5:2 resonance}

Since we are interested in the Pallas-Phaethon transportation route, we calculate the FLI map of the 5:2 MMR with Jupiter in the orbital plane of Pallas, that is for $i=34.84 \mathrm{deg}, \Omega=173 \mathrm{deg}, \omega=310 \mathrm{deg}$ and $M=249 \mathrm{deg}$, using $500 \times 500$ test particles with semi-major axis and eccentricity in between $a \times e=[2.805,2.838] \times[0,0.589]$. This map, computed for $5 \mathrm{Kyrs}$, is given in Figure 1. The color scale, adjusted to FLI values is chosen such that stable particles with $F L I<0.9$ are red, while the more chaotic ones with FLIs larger than 1.1 are yellow.

All our computations are performed using the ORBIT9 integrator $^{1}$ and the calculation time for one map is around 15 minutes $^{2}$. More technical details about the computations of the maps can be found in Todorović (2017).

The $(a, e)$ resonant profile visible in Fig. 1 has an hourglass structure, different from the typical V shape of a mean motion resonance. Other high-order resonances are also visible herein, but unlike the usual straight vertical $\mathrm{V}$ shaped resonant forms, those resonances are also deviated in the region of the unstable point. The unstable point (narrowest part of the resonance) is displaced to large eccentricity, close to $e \sim 0.3$, where we can notice a peculiar deviation of the separatrix.

For a better illustration, an enlarged portion of Fig. 1 close to the unstable point, in the region $[a, e]=$ $[2.814,2.827] \times[0.190,0.364]$ is plotted on the Fig.2. The deviated shapes of the separatrix remind strongly on the socalled hyperbolic invariant manifolds of the saddle point of the resonance (Morbidelli 2002), the crucial elements in the production of chaos. Let us mention that invariant manifolds are identified as sources of natural transportation routes, and are therefore very important in space trajectory design and astrodynamics in general (Gomez et al 2004; Vilac 2008; Belló et al 2010). Their localization however, is

1 Available from http://adams.dm.unipi.it/orbfit/.

2 We use the Fermi cluster at the Astronomical Observatory of Belgrade. The cluster has 12 worker nodes, each node (HP SL390S blade server G7 X5675) has a $2 \times X 5675$ (2x6core) processor, $3.1 \mathrm{GHz}, 24 \mathrm{G}$ memory, $2 \mathrm{~Tb}$ disc and 2xM2090 NVIDIA Teslafermi GPU cards.

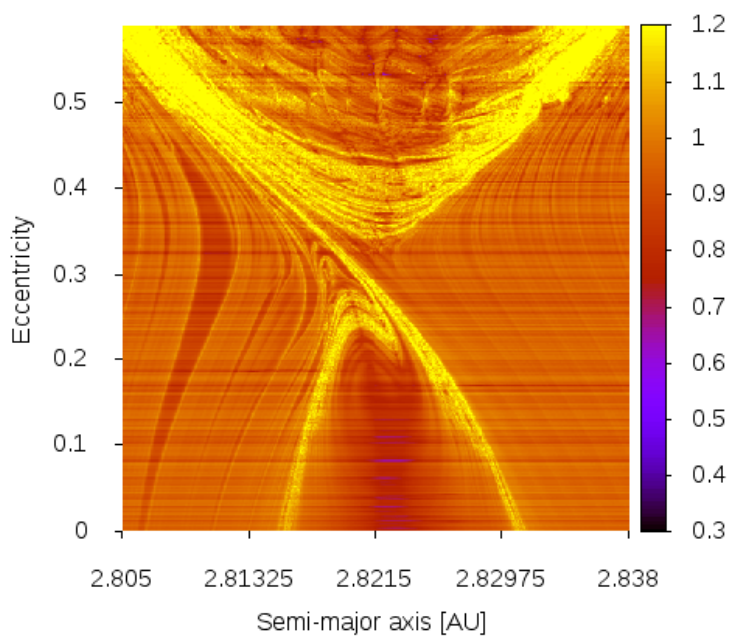

Figure 1. The 5:2 mean motion resonance with Jupiter computed in the orbital plane of Pallas, for $5 \mathrm{Kyrs}$ and for $a \times e=$ $[2.805,2.838] \times[0,0.589]$. Other orbital elements are fixed to $i=34.80 \mathrm{deg}, \Omega=173.09, \omega=310$ and $M=248.97$. Stable particles are red, while the most chaotic ones are yellow. The $(a, e)$ profile of the resonance has an atypical hourglass structure and the location of the unstable point is unusually high (close to $e \sim 0.3)$.

not trivial, even in highly restricted dynamics. Algorithms for their numerical evaluation can be found for example in Simo (1999); Koon (2011); Topputo (2016). Treating a circular restricted three-body problem, Guzzo and Lega (2014) illustrated that invariant manifolds can be captured also by short-term FLI maps. Using this ability of FLI and a real Solar System model ${ }^{3}$ in Todorović (2017) we detected hyperbolic manifolds-like structures in the 5:2 MMR with Jupiter, and we illustrated that such structures are fruitful sources of fast diffusing orbits. We have also observed that 8 per cent of test bodies placed into the 5:2 MMR recovered a Phaeton like orbit.

Following the same methodology as in Todorović (2017), but focusing on the dynamical pathways between Phaethon and Pallas, we change the initial orbital plane inside the 5:2 MMR, as described above. Then, we chose 1000 of the most chaotic particles in between $e \in[0.19,0.364]$ along the intricate yellow structures on the Fig. 2. An eventual injection of Pallas fragments into the 5:2 MMR fits well in the chosen range in eccentricity, since the eccentricity of Pallas is $\sim 0.23$ and the remaining Pallas family members have $0.255<e<0.283$. We follow their orbital evolution for 5 Myrs and count all the bodies that at some moment recovered the orbit of Phaethon. That is, we search in the $(a, e, i)$ element space those particles that satisfy

$\left|a-a_{P h}\right|<0.1,\left|e-e_{P h}\right|<0.1,\left|i-i_{P h}\right|<3$,

3 We treat all planets from Venus to Neptune, while the mass of Mercury is added to the mass of the Sun and the corresponding barycentric correction to initial conditions is applied. 


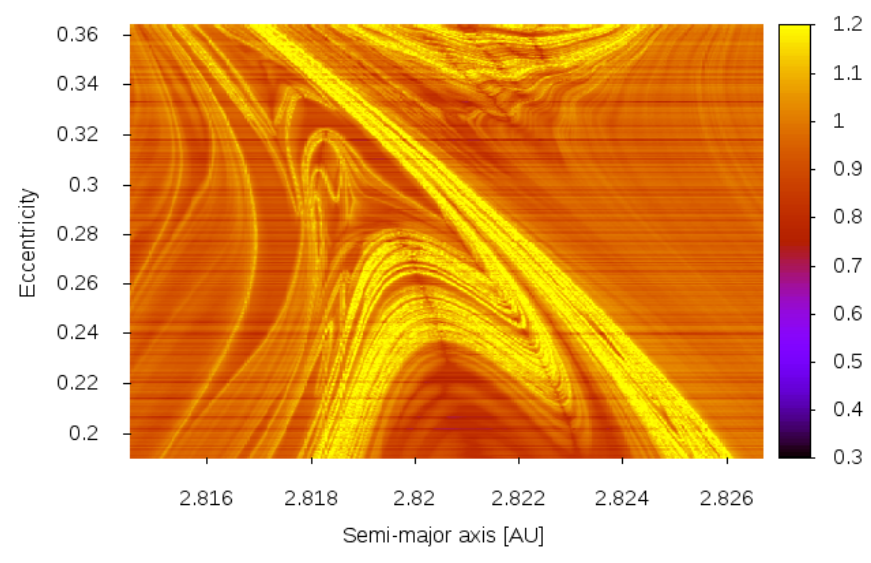

Figure 2. The enlarged portion of the 5:2 MMR in the vicinity of the unstable point, where we can notice the peculiar deviation of the separatrix. Such shapes look very similar to the traces of the normally hyperbolic invariant manifolds. The candidates for diffusion are selected along the yellow structures. The range in eccentricities $e \in[0.19,0.364]$ includes the eccentricity of Pallas and other family members of the Pallas family.

where $\left(a_{P h}, e_{P h}, i_{P h}\right)$ are the respective semi-major axis, eccentricity and inclination of Phaethon. These elements are taken from the AstDys database ${ }^{4}$ and their osculating values for the epoch 57600.0 MJD are $a_{P h}=1.271 \mathrm{AU}, e_{P h}=0.889$ and $i_{P h}=22.24 \mathrm{deg}$.

Mapping the resonance and selecting particles in the orbital plane of Pallas, strongly affected the number of bodies reaching Phaeton. Instead of 8 per cent observed in Todorović (2017), we found that among 1000 test bodies placed into the 5:2 MMR, 436 objects satisfy the condition (1). The first fragments placed into the resonance arrived to the current position of Phaethon after 0.294 Myrs and their median delivery time is 1.7 Myrs.

\subsection{The 8:3 resonance}

The map of the 8:3 MMR computed in the orbital plane of Pallas is illustrated in Figure 3. The FLIs are calculated for $5 \mathrm{Kyrs}$ and for $[a, e]=[2.696,2.714] \times[0,0.4]$, while the other orbital and numerical parameters are the same as those for Fig. 1. Contrary from the 5:2 MMR, this resonance does have the standard V shape, the map is depleted from peculiar structures, and chaos manifests only along the resonant border. Neither the unstable point or high-order neighboring resonances are visible on the map.

Again searching for Phaethon-like orbits, we selected 1000 particles along the separatrix in the eccentricity range $e \in[0.2,0.36]$ and repeated the same procedure as described above: we integrate the objects for 5 Myrs and count all the particles entering into the domain given in (1).

The 8:3 MMR delivered slightly more bodies to the current position of Phaethon than the 5:2 MMR, in total 469,

4 Available at http://hamilton.dm.unipi.it/astdys/.

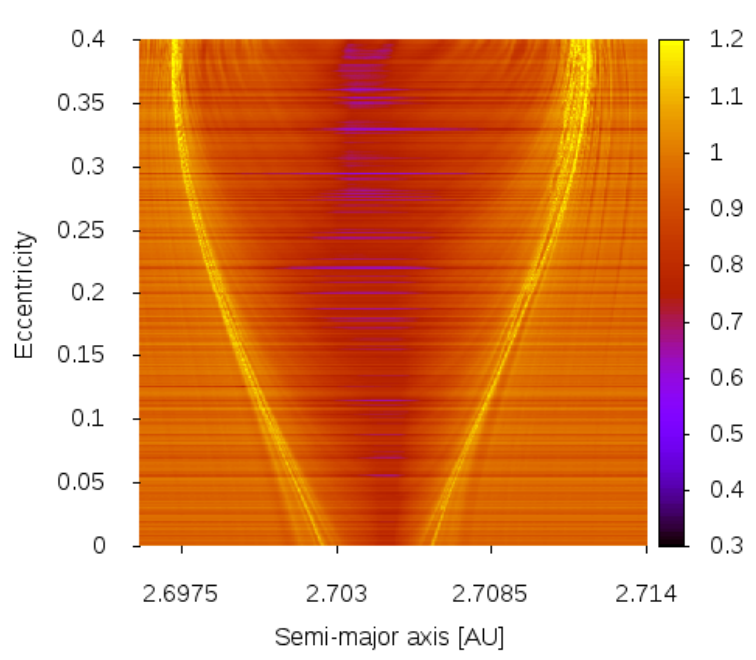

Figure 3. The FLI map of the $8: 3$ mean motion resonance with Jupiter computed for $5 \mathrm{Kyrs}$ in the range $[a, e]=[2.696,2.714] \times$ $[0,0.4]$. Other numerical parameters are the same as for Fig. 1. The particles for diffusion, candidates for reaching the orbit of Phaethon are selected along the resonant border in between $e \in$ $[0.2,0.36]$.

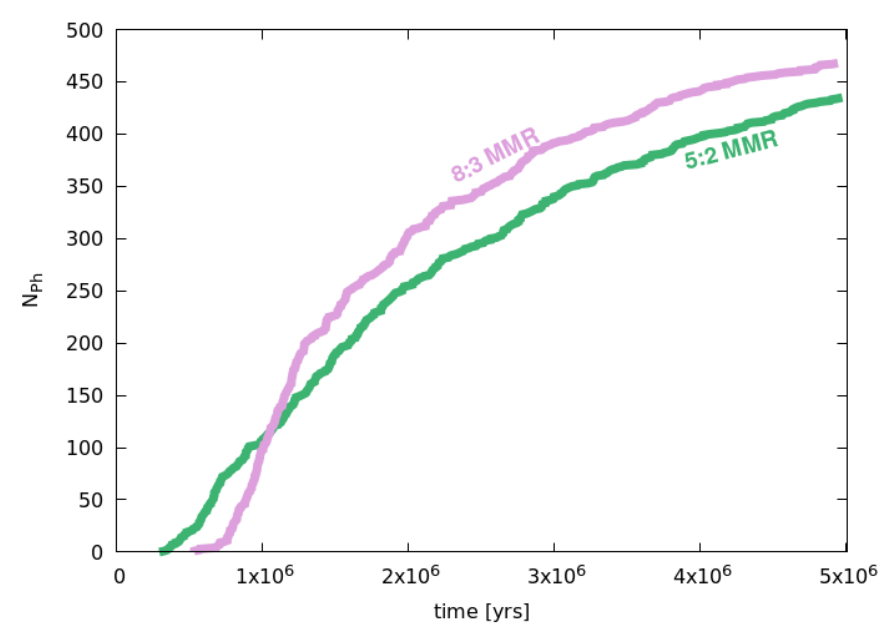

Figure 4. The green line shows the number of particles migrating from the 5:2 MMR into the neighborhood of Phaethon and the pink line gives the same for the 8:3 MMR. Both resonances show similar transportation abilities. The 5:2 MMR delivered 436 bodies to the neighborhood of Phaethon, and the 8:3 MMR was a little more efficient in that respect with 469 objects. The first arrivals to the destination are detected after 294500 and 532500 years, respectively.

with the first arrivals occurring after 0.53 Myrs. The median transportation time along this route is 1.5 Myrs.

Figure 4 shows the influx rates of particles originating from the 5:2 (green) and 8:3 MMRs (pink). In the first million years, the 5:2 MMR delivered more particles towards Phaethon; yet, in the following Myrs the 8:3 MMR took over 
the transport role. With 43.6 and 46.9 per cent of test bodies delivered to Phaethon, the two resonances show similar transfer abilities, a result different from the one obtained in de Leon et al (2010), who found that the 8:3 MMR was three times more efficient in this respect than the 5:2 resonance. However, the result in de Leon et al (2010) was based on a smaller sample (only 2 per cent) and the integration times were much longer, 100 Myrs.

Both Phaethon and Pallas, source and destination region of our test particles, are in highly inclined orbital planes, which certainly affects the amount of material delivered along the route.

\section{CONCLUSION}

We have shown that the 5:2 and 8:3 MMRs with Jupiter possess a very powerful mechanism for transporting objects to the near-Earth region close to the asteroid Phaethon. The results we obtained are significantly different than earlier studies. In the work of de Leon et al (2010), where both 5:2 and 8:3 MMRs where studied the probability for this connection was 2 per cent, and in Bottke et al (2002) for example, this probability was zero. In Todorović (2017) we applied the same methodology as done here and found that 8 per cent of test objects placed into the 5:2 MMR reached the orbit of Phaethon; much less than in this work, because, in Todorović (2017), the resonance was mapped in the lower inclined orbital plane of Ceres (at $i=10 \mathrm{deg}$ ). Choosing particles at the most unstable parts of the two resonances, but in the orbital plane of Pallas increased the Pallas-Phaethon transportation efficiency multiply times. Namely, almost half of test objects placed in the two resonances migrated to the neighborhood of Phaethon, strongly supporting the hypotheses of the Pallas origin of Phaethon and the Geminids meteor showers.

\section{ACKNOWLEDGEMENTS}

This research was supported by the Ministry of Education, Science and Technological Development of the Republic of Serbia, under the project 176011 'Dynamics and kinematics of celestial bodies and systems'. The calculations were performed on a Fermi cluster located at the Astronomical Observatory of Belgrade, purchased by the project III44002 'Astroinformatics: Application of IT in astronomy and close fields'. The author is grateful to A. Rosengren and the two reviewers for useful remarks.

\section{REFERENCES}

Belló, M., Gómez, G., Masdemont, J.J., 2010, Space Manifold Dynamics, Springer, New York, 1-96

Bottke, W., Morbidelli, A., Jedicke, R., Petit, J-M., Levison, H., Michel, P. and Metcalfe, T., 2002, Icarus, 156, 399-433

Daquin, J., Rosengren, A.J., Alessi, E.M., Deleflie, F., Valsecchi, G. and Rossi, A., 2016, Celest. Mech. Dynam. Astron.,124, 4, 335

Delbo, M., Libourel, G., Wilkerson, J., Murdoch, N., Michel, P., Ramesh, K., Ganino, C., Verati, V., Marchi, S., Bottke, W., Morbidelli, A., Jedicke, R., Petit, J-M., Levison, H., Michel, P. and Metcalfe, T., 2014, Nature, 508, 233 de León, J., Campins, H., Tsiganis, K., Morbidelli, A. and Licandro, J., 2010, A\&A, 513, A26

DeMeo, F. E. and Binzel, R. P. and Slivan, S. M. and Bus, S. J., 2009, Icarus, 202, 160

Dvorak R., Pilat-Lohinger E., Funk B., Freistetter F, 2003, A\&A, 398, L1

Froeschlé, Cl., Gonczi, R. and Lega, E., 1997, Planetary and Space Science, 45, 881

Froeschlé, Cl., Lega, E. and Gonczi, R.,1997, Celest. Mech. Dyn. Astron., 67, 41

Froeschlé, C.,Guzzo, M. and Lega, E., 2000, Science, 289 N.5487:2108

Froeschlé, C., Guzzo, M. and Lega, E., 2005, Celest. Mech. Dynam. Astron., 92, 243

Gómez, G., Koon, W. S., Lo, M. W., Marsden, J. E., Masdemont, J. and S D Ross, 2004 Nonlinearity, 17, 1571

Green, S. and C. Kowal, C., 1983, IAU Circular No. 3878

Gustafson, B. A. S., 1989, A\&A , 225, 533

Guzzo, M., 2005, Icarus, 174, 273

Guzzo, M., 2006, Icarus, 181, 475

Guzzo M. and Lega E., 2014, SIAM J. Appl. Math., 74, 4, 1058

Hanuš, J., Delbó, M., Vokrouhlický D., Pravec, P., Emery, J. P., Alí-Lagoa, V., Bolin, B., Devogèle, M., Dyvig, R., Galád, A., Jedicke, R., Kornoš, L., Kušnirák, P., Licandro, J.,Reddy, V., Rivet, J.-P., Világi, J. and Warner, B. D., 2016, Astronomy \& Astrophysics, 592, A34

Jenniskens, P., 1994, Astronomy and Astrophysics, 287, 990

Jewitt, D., Li, J. and Agarwal, J.,2013, The Astrophysical Journal Letters, 771, L36

Koon, W.S., Lo, M.W., Marsden, J.E. and Ross, S.D., 2011 Marsden Books, ISBN 978-0-615-24095-4.

Lega, E. and Froeschlé, C., 2003, Phys D, 182, 179

Morbidelli, A., 2002, Modern celestial mechanics: aspects of solar system dynamics, Taylor \& Francis

Pilat-Lohinger, E. and Dvorak, R., 2002, Celest. Mech. Dynam. Astron. 82, 143

Rosengren, A., Daquin,J., Alessi, E. M., Deleflie, F., Rossi, A., Valsecchi, G. B., 2015, arXiv:1512.05822

Sándor, Z., Suli, A., Erdi, B., Pilat-Lohinger, E., Dvorak, R., 2007, Mon.Not.R.Astron.Soc.375, 1495

Schwarz, R., Haghighipour, N., Eggl, S., Pilat-Lohinger, E. and Funk, B., 2011, Mon. Not. R. Astron. Soc. 414, 2763-2770

Simo, C., 1999, NATO ASI Ser. C Math. Phys. Sci., Dordrecht. Kluwer Acad. Publ., 533, 223

Todorović, N., Lega, E., Froeschlé, C., 2008, Celest. Mech. Dynam. Astron., 102, 13

Todorović, N., Guzzo, M., Lega, E. and Froeschlé, C., 2011, Celest. Mech. Dynam. Astron., 110, 389

Todorović, N. and Novaković, B., 2015, MNRAS, 451, 1637

Todorović, N., 2017, Mon. Not. R. Astron. Soc., 465, 4441

Topputo, F., 2016, Communications in Nonlinear Science and Numerical Simulation, 32, 89

Villac, B. F., 2008, Celest. Mech. Dynam. Astron., 102, 29

Whipple, F. L., 1983, IAU Circ., 3881

Williams, I.P. and Wu, Z., 1993, Mon. Not. R. Astron. Soc., 262, 231 